9 - ORIGINAL ARTICLE

PLASTIC SURGERY

\title{
Botulinum toxin type A on cutaneous flap viability in diabetic and tobacco-exposed rats ${ }^{1}$
}

\author{
Cristina Pires Camargo ${ }^{\mathrm{I}}$, Alfredo Luiz Jacomo ${ }^{\mathrm{II}}$, Cláudia Naves Battlehner ${ }^{\mathrm{III}}$, Miriam Lemos ${ }^{\mathrm{IV}}$, Paulo Hilário Saldiva ${ }^{\mathrm{III}}$, Milton \\ Arruda Martinsv, Alexandre Mendonça Munhoz ${ }^{\mathrm{VI}}$, Rolf Gemperli ${ }^{\mathrm{VII}}$
}

DOI: http://dx.doi.org/10.1590/S0102-865020150090000009

IFellow PhD degree, Postgraduate Program in Surgical Clinics, Plastic Surgery Division, Faculty of Medicine, Universidade de São Paulo (FMUSP), Brazil. Scientific and intellectual content of the study, interpretation of data, critical revision.

IIPhD, Department of Anatomy, FMUSP, Sao Paulo-SP, Brazil. Interpretation of data, critical revision.

"IIPhD, Department of Pathology, FMUSP, Sao Paulo-SP, Brazil. Interpretation of data, critical revision.

Iv PhD, Department of Anatomy, FMUSP, Sao Paulo-SP, Brazil. Technical procedures, acquisition and interpretation of data.

${ }^{v} \mathrm{PhD}$, Department of Internal Medicine, FMUSP, Sao Paulo-SP, Brazil. Interpretation of data, critical revision.

${ }^{\mathrm{VI}} \mathrm{PhD}$, Assistant Professor, Institute of Research, Hospital Sírio-Libanês, Sao Paulo-SP, Brazil. Interpretation of data, critical revision.

${ }^{V I I}$ Full Professor and Head, Plastic Surgery Division, Department of Surgery, FMUSP, Sao Paulo-SP, Brazil. Scientific and intellectual content of the study, interpretation of data, critical revision.

\begin{abstract}
PURPOSE: To investigate the effect of Botulinum toxin A (BoNTA) on skin flap viability in healthy, tobacco-exposed and diabetic rats. METHODS: Ninety male Wistar rats (250-300g) were randomly divided into six groups: control+saline (C1), control+BoNTA (C2), tobacco-exposed+saline (T1), tobacco-exposed+BoNTA (T2) diabetes+saline (D1) and diabetes+BoNTA (D2). A dorsal cutaneous flap $(3 \times 10 \mathrm{~cm})$ was performed. Survival area and total area of the flaps were measured. Lumen diameter, external arterial diameter and lumen/wall thickness ratio were recorded.

RESULTS: Survival area increased in control group with BoNTA injection compared with control animals injected with saline (C2 $\mathrm{x}$ $\mathrm{C} 1 ; 0.9 \pm 0.1$ vs $0.67 \pm 0.15, \mathrm{p}=0.001)$. A similar result was found in diabetes group injected with BontA (D2 x D1; $0.97 \pm 0.2 v s 0.61 \pm 0.24$, $\mathrm{p}=0.018)$. No difference was observed in skin flap viability in tobacco-exposed groups (T2 $\mathrm{T} 1 ; 0.74 \pm 0.24 v s 0.64 \pm 0.21, \mathrm{p}=0.871)$. Lumen diameter $(p=0.004)$, external arterial diameter $(p=0.0046$, ) and lumen/wall thickness ratio $(p=0.003)$ were increased in diabetes+BoNTA-treated animals. This effect was not observed in control or in tobacco-exposed groups.
\end{abstract}

CONCLUSIONS: Botulinum toxin A increased skin flap viability in control and diabetic rats on the seventh post-operative day. Increased lumen diameter, external arterial diameter, and lumen/wall thickness ratio were observed in the diabetes+BoNTA group. BoNTA had no effect in the tobacco-exposed group on the seventh postoperative day.

Key words: Surgical Flaps. Botulinum Toxins, Type A. Diabetes Complications. Tobacco. Rats. 


\section{Introduction}

Skin flap procedures are frequently used in plastic surgery to repair local tissue defects. Among the main techniques, random flaps are well described and widely performed in reconstructive surgery. In spite of the advantages, random flaps are not reliable and due to the random-pattern distribution of skin vascularization, the technique can be limited by a low blood supply to the subdermal and dermal vascular plexuses. In fact, the distal aspect of a random pattern flap has a high risk of ischemic necrosis, which often results in partial loss of the flap, requiring further reconstructive procedures ${ }^{1}$.

Extrinsic factors increase the risk of flap necrosis. Structural and functional problems in skin microcirculation of diabetic and smoker patients reduce skin perfusion. In fact, diabetes causes endothelial dysfunction, increased oxidative stress, and decreased epidermal cell migration, which hinders the wound healing process ${ }^{2,3}$. Tobacco exposure causes vasoconstriction, increasing the risk of flap necrosis ${ }^{4}$.

Procedures aimed at improving flap survival have been the subject of intensive research. Improvement in clinical risk factors, including smoking cessation and control of diabetes, are well established. However, modalities that specifically target the flap have shown mixed results ${ }^{5-7}$. Experimental models of skin flap necrosis have been employed to study the clinical conditions that increase risk $^{8}$ as well as to evaluate strategies that improve flap survival, such as vasodilators ${ }^{9-11}$. Nevertheless, few studies have evaluated the effect of botulinum toxin type A (BoNTA) in skin flap viability. These studies showed a positive effect in cutaneous flap viability in healthy rats ${ }^{12,13}$. However, there are no studies dealing with the effect of BoNTA in diabetic and tobacco-exposed animals.

BoNTA acts on the neuromuscular junction in striated muscle ${ }^{14}$. Recent studies have suggested that BoNTA also has a vasodilation effect probably by autonomic nerve block ${ }^{14-16}$. It is hypothesized that BoNTA acts by blocking norepinephrine and other vasoconstriction substances in the neuromuscular junction in the arterial smooth muscle, thus promoting vasodilation and consequently increasing blood supply in the flap.

In cutaneous flaps, the most important survival factor is dermic and subdermal plexus blood flow. Previous studies only showed the effect of BoNTA in healthy animals. Due to the high incidence of diabetes and tabagism in the population and the impairment in vascularization in these two conditions, investigating the effect of BoNTA on cutaneous flap viability seemed important. This study aimed to determine the potential benefit of BoNTA on cutaneous flap survival using rats submitted to standard skin flap procedures. For this purpose, dorsal cutaneous flaps, treated with either saline solution or BoNTA, were evaluated in healthy, diabetic, and tobacco-exposed animals.

\section{Methods}

The protocol was approved by the Ethics Committee of the Universidade de São Paulo (110/2011). All procedures strictly followed current regulations related to animal experimentation dictated by the Council for International Organization of Medical Sciences ethical code for animal experimentation.

Ninety male Wistar rats (weight: 250-300g) were distributed into six groups: control+saline $(\mathrm{C} 1)$, control+BoNTA (C2), diabetes+saline (D1), diabetes+BoNTA (D2), tobaccoexposed + saline (T1), and tobacco-exposed+BoNTA (T2). All animals were housed at the Laboratory of Microsurgery and Plastic Surgery (LIM-04) at USP, under controlled night/day cycle, temperature, and humidity conditions. Standard rat chow and water were available ad libitum.

\section{Induction of diabetes}

Streptozotocin (streptozotocin mixed anomers 031M1287V, Sigma Chemical, St. Louis, MO) was injected via the penile vein under inhaled anesthesia (20\% isoflurane; 150 $200 \mathrm{ml} / \mathrm{min}$ ). A total of $55 \mathrm{mg} / \mathrm{kg}$ of streptozotocin diluted in phosphate-buffed saline ( $\mathrm{pH} 4$ ) was used. Glycemic levels were checked before and 24 hours after injection to confirm diabetic induction ${ }^{18}$. The animals were then maintained for eight weeks and subsequently randomized to receive BoNTA or saline solution. Since this eight-week period was necessary for the diabetic animals, the other groups were maintained, treated and euthanized at the same age.

\section{Tobacco exposure}

The animals were exposed in a 28-L plastic box with three orifices: one inlet for synthetic air ( $2 \mathrm{~L} / \mathrm{min})$; another for smoke; and an outlet to ventilate excess smoke. The smoke inlet was connected to a Venturi system controlled by means of fluorometry (2.5 L/min), which in turn was connected to a lit cigarette.

Carbon monoxide (CO) was monitored using a single gas detector (ToxiPro; Biosystems, USA) to maintain a $\mathrm{CO}$ concentration of 300-350 ppm (parts per million) inside the box. The tobacco exposure schedule consisted of two 30-minute 
sessions per day for 28 consecutive days. After 14 days of tobacco exposure, the animals received BoNTA or saline injection. One week later the surgery was performed. The 28-day tobacco exposure scheme ended at the day of sacrifice.

\section{Saline solution and BoNTA groups}

A rectangular cutaneous area $(3 \times 10 \mathrm{~cm})$ was bounded with permanent pen marker on the dorsal region. Along the future flap line, 2 rows of 10 puncture points ( $1 \mathrm{~cm}$ apart) were intradermally injected with $0,02 \mathrm{ml} \mathrm{BoNTA} /$ puncture, total of $20 \mathrm{u} /$ rat $\left(\right.$ Botox ${ }^{\circledR} 100 u$, Allergan. Irvine. CA. USA) (C2, D2, T2) or with $0.02 \mathrm{ml}$ saline/puncture (C1, D1, T1) (Figure 1). Seven days later, surgery was performed.

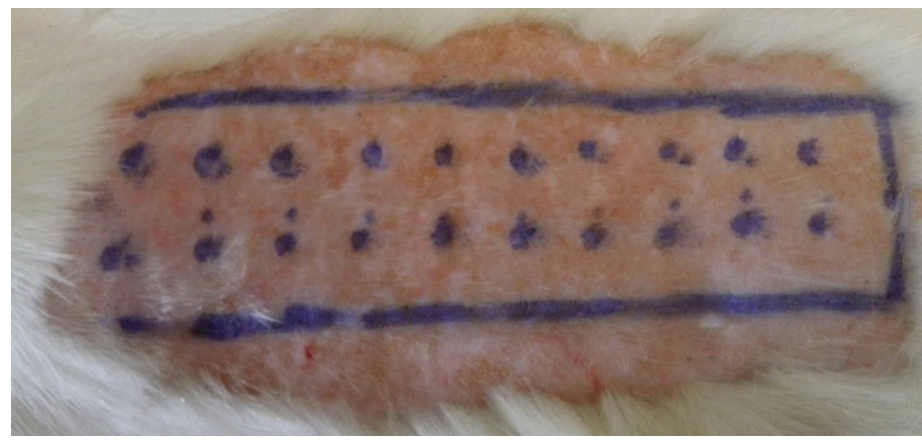

FIGURE 1 - The scheme of BoNTA or saline injection in the cutaneous flap.

\section{Surgical procedure}

Antisepsis was performed using $0.5 \%$ chlorhexidine. Under inhaled anesthesia (20\% isoflurane; $150-200 \mathrm{ml} / \mathrm{min}$ ) the $3 \times 10 \mathrm{~cm}$ cranial flap based on the scapulae was elevated. The flap was then returned to its original position and sutured using separate stitches of 4-0 mononylon. Seven days after surgery, the rats were euthanized by anesthetic overdose (pentobarbital sodium $200 \mathrm{mg} / \mathrm{kg}$ ). Weight and glycemia were measured.

\section{Skin flap necrosis evaluation}

Immediately before euthanasia the flaps were photographed (Olympus $3.5 \mathrm{~mm}$ digital camera; Olympus Stylus ${ }^{\circledR}$, Japan) with a ruler along the length of the flap. Total area of the flap and area of necrosis were measured (squared millimeters) using the Image ${ }^{\circledR}$ software (NIH; http://rsbweb.nih.gov/ij). Viable skin was defined as pink, warm, and soft to touch. Necrotic areas were defined when brown-to-black, cold, and hard to touch (Figure 2). The percentage of area of healthy skin (survival area) was then calculated.

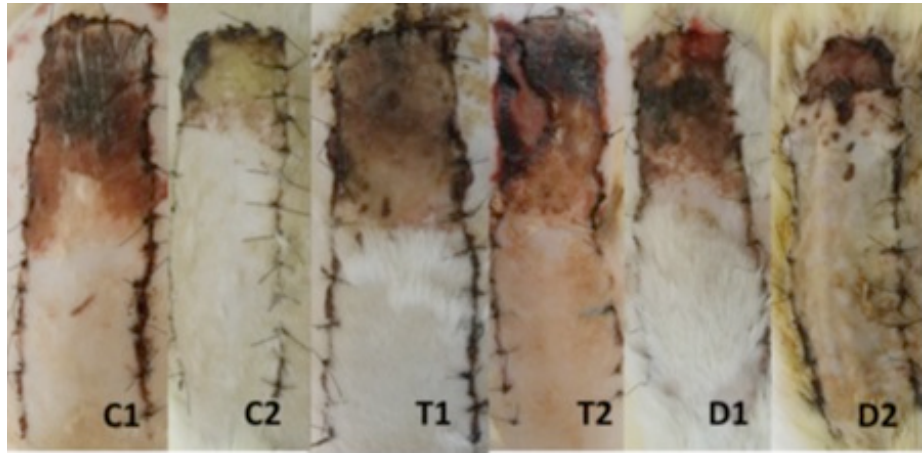

FIGURE 2 - Dorsal cutaneous flaps of rats at the day of euthanasia. C1control+saline; C2- control+BoNTA; T1- tobacco-exposed+saline; T2tobacco exposed+BoNTA; D1- diabetes+saline; D2- diabetes+BoNTA.

\section{Microscopic evaluation}

Immediately after death, a skin rectangle exceeding in $1.5 \mathrm{~cm}$ the limits of the flap was harvested, carefully extended on filter paper to avoid torsion and immersed in fixative (4\% neutral, phosphate buffered, paraformaldehyde), for 48 hours at room temperature. A long stripe of tissue, $0.5 \mathrm{~cm}$ wide, was then cut along the major axis of the flap, including the longest extension of necrosis in the cranial direction for each case. Both its ends were marked with Indian ink and the stripe cut in the middle. The fragments were identified as "cranial" and "caudal". Tissue was washed, dehydrated in graded concentrations of alcohol and embedded in paraffin. Four-micrometer-thick sections were mounted on glass slides and stained with hematoxylin and eosin. The sections were analyzed under the light microscope for description of the skin. The diameter of all arteries pertaining to the deep vascular plexus of the dermis was measured in images obtained by an image analysis system formed by a Nikon Optiphot-2 (Nikon, Tokio, Japan) microscope, coupled to a Nikon DXM $1200 \mathrm{~F}^{\circledR}$ (Nikon, Tokio, Japan) video digital camera. Measurements were performed using the Image-Pro Plus ${ }^{\circledR}$ program version 6.0 (Media Cybernetics, Inc, Silver Spring, MD, USA) as follows: for each arterial profile, independently of the section direction (transversal or oblique to the lumen), it was first traced a line over the major axis of the profile. A second line, perpendicular to the previous, delimiting the largest possible external diameter of the vessel was traced. The length of the second line corresponds to the arterial diameter (in micrometers $)^{24,25}$. Guided by the second line, the thickness of the arterial wall was measured in the two sites where the line crossed the wall (Figure 3). 

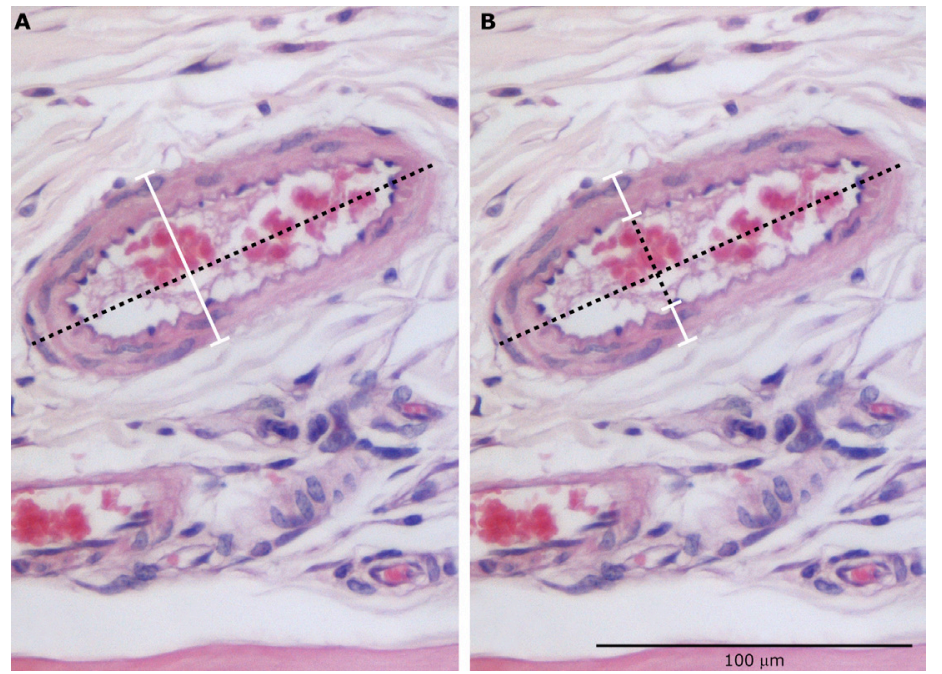

FIGURE 3 - Oblique section of an artery of the deep vascular plexus of rat skin. A) The traced line was drawn along the major axis of the profile; the white line is perpendicular to the first and delimits the largest external diameter of the artery. B) Two measurements were taken of the thickness of the arterial wall, from the endothelial surface to the adventitia, guided by the "diameter" line.

For statistical analysis, the parameters external diameter of the arteries, mean thickness of the arterial wall (arithmetic mean between the two measurements for each profile), lumen diameter (external diameter minus (2x mean thickness for each profile), and Lumen/Wall Thickness (lumen diameter divided by mean thickness for each profile) were calculated and recorded in micrometers with exception of the ratio.

\section{Statistical analysis}

For area evaluations, the Mann-Whitney test was applied; for the other data, comparing multiple groups, Analysis of Variance (ANOVA) was used. The post hoc test of Tukey was employed for the pairwise comparisons. All analyses were performed using IBM SPSS statistical software (Statistical Package for the Social Sciences), version 21.0. The statistical significance level was set at 5\%.

\section{Results}

Two animals in the diabetic group died. None of the animals of the BoNTA groups presented systemic effects such as weakness or respiratory distress.

\section{Area evaluation}

Both control (C2 x C1; $0.9 \pm 0.1$ vs. $0.67 \pm 0.15$, respectively) and diabetic (D2 x D1; $0.97 \pm 0.2$ vs. $0.61 \pm 0.24$, respectively) groups showed a statistically significant enlargement of the survival area when BoNTA was injected (Controls, $\mathrm{p}=0.001$; Diabetics, $\mathrm{p}=0.018$ ) (Figure 2). No statistically significant difference was observed in skin flap viability in tobacco-exposed groups (T2 x T1; $0.74 \pm 0.24$ vs. $0.64 \pm 0.21, \mathrm{p}=0.871)$.

\section{Microscopic evaluation}

When the groups were compared for the parameters: lumen diameter, external arterial diameter, and lumen/wall thickness ratio, the ANOVA resulted $\mathrm{p}=0.001 ; \mathrm{p}=0.001 ; \mathrm{p}=0.04$, respectively. The pairwise comparisons are shown in Tables 1-3. Only among diabetic animals, statistically significant differences were observed; with the BoNTA injected group presenting larger diameters of arteries and lumens, and lumen/wall thickness ratio than diabetes+saline group. No significant difference was detected when wall thickness of the arteries was compared.

TABLE 1 - Pairwise comparisons between the groups in relation to the lumen diameter (Tukey test).

\begin{tabular}{ccccc}
\hline & & $\begin{array}{c}\text { Mean } \\
\text { (C1/C2) }\end{array}$ & $\begin{array}{c}\text { SD } \\
(\text { C1/C2 })\end{array}$ & $p$ \\
\hline Saline & BoNTA & & & \\
C1 & C2 & $24.21 / 27.16$ & $19.39 / 17.94$ & 0.44 \\
T1 & T2 & $26.60 / 35.59$ & $20.70 / 20.52$ & 0.762 \\
D1 & D2 & $21.50 / 30.18$ & $12.02 / 21.56$ & 0.004 \\
\hline
\end{tabular}

C1- control+saline; C2- control+BoNTA; T1- tobacco-exposed+saline; T2- tobacco exposed+BoNTA; D1- diabetes+saline; and D2- diabetes+BoNTA; SD- Standard Deviation.

TABLE 2 - Pairwise comparisons between the groups in relation to the external arterial diameter (Tukey test).

$\begin{array}{ccc}\text { Mean } & \text { SD } & p \\ (\mathrm{C} 1 / \mathrm{C} 2) & (\mathrm{C} 1 / \mathrm{C} 2)\end{array}$

Saline BoNTA

$\begin{array}{lllll}\text { C1 } & \text { C2 } & 43.30 / 46.36 & 21.51 / 19.02 & 0.298 \\ \text { T1 } & \text { T2 } & 43.04 / 41.08 & 22.51 / 22.18 & 0.754 \\ \text { D1 } & \text { D2 } & 38.85 / 46.47 & 14.87 / 25.58 & 0.0046\end{array}$

C1- control+saline; C2- control+BoNTA; T1- tobacco-exposed+saline; T2- tobacco exposed+BoNTA; D1- diabetes+saline; and D2- diabetes+BoNTA; SD- Standard Deviation.

TABLE 3 - Pairwise comparisons between the groups in relation to lumen/wall thickness ratio (Tukey test).

\begin{tabular}{ccccc}
\hline & & $\begin{array}{c}\text { Mean } \\
(\mathbf{C 1} / \mathbf{C} 2)\end{array}$ & $\begin{array}{c}\text { SD } \\
(\mathbf{C 1} / \mathbf{C} 2)\end{array}$ & $\boldsymbol{p}$ \\
\hline Saline & BoNTA & & & \\
$\mathrm{C} 1$ & $\mathrm{C} 2$ & $3.20 / 3.07$ & $2.27 / 2.18$ & 0.94 \\
$\mathrm{~T} 1$ & $\mathrm{~T} 2$ & $3.43 / 3.45$ & $2.65 / 2.56$ & 0.941 \\
$\mathrm{D} 1$ & $\mathrm{D} 2$ & $2.69 / 4.05$ & $2.69 / 2.70$ & 0.003 \\
\hline
\end{tabular}

C1- control+saline; C2- control+BoNTA; T1- tobacco-exposed+saline; T2- tobacco exposed+BoNTA; D1- diabetes+saline; and D2- diabetes+BoNTA; SD- Standard Deviation. 


\section{Discussion}

Skin flaps based on random blood supply are frequently indicated for the surgical treatment of skin and soft tissue defects, but are at high risk for ischemia and distal necrosis due to low axial blood flow ${ }^{1,7,8}$. In fact, tissue ischemia is a postoperative complication that can cause partial or full thickness skin flap necrosis with unsatisfactory functional and aesthetic outcome ${ }^{4}$. In random pattern skin flaps, partial distal flap necrosis is difficult to avoid and treat. Thus, in order to improve the survival rate of skin random flaps, considerable efforts have been done to increase blood flow and to improve its tolerance to ischemia ${ }^{5,6,9}$. In recent years, surgical techniques and pharmacologic substances that can improve blood supply and in this manner increase flap survival were tested and could provide a valuable method for minimizing flap necrosis. Surgical delayed technique is a suitable procedure, but requires additional interventions and is more invasive. Previous experimental and clinical studies have demonstrated that systemic and/or local drug agents can help overcome tissue ischemia and skin flap necrosis by inducing angiogenesis ${ }^{12,13}$, vasodilatation ${ }^{9,10}$ or decreasing tissue damage caused by ischemia ${ }^{6}$.

Botulinum toxin type A is commonly used for aesthetic and reconstructive objectives worldwide, as it is a blocker of acetylcholine release at the neuromuscular junction ${ }^{14}$. In fact, the effect of muscle relaxation has made it helpful in the management of hemifacial spasm, strabismus, and blepharospasm ${ }^{14}$. Botulinum toxin type A has been recently studied as a vasodilator agent in experimental and clinical studies as this neurotoxin has also been successfully used in the management of Raynaud's phenomenon and preventive on vasospasm following microvascular anastomosis $^{11-13}$. Two mechanisms have been described for the inhibitory effect of BoNTA on vasospasm: inhibition of sympathetically mediated vasospasm in cold environment and prevention of vascular smooth muscle receptor recruitment ${ }^{15,16}$. In addition, it is hypothesized that this neurotoxin could inhibit the release of the neurotransmitter at the vascular neuromuscular junction by blocking the presynaptic release of acetylcholine ${ }^{8}$.

Preventive effect of BoNTA on skin flap necrosis has been reported in only few experimental studies ${ }^{11-13}$. Its use on other clinical conditions, such as vascular diseases, remains unclear. Additionally, there are no previous controlled experimental studies concerning the effect of BoNTA on diabetic and tobacco-exposed animals submitted to random skin flaps.

In the present study, we focused on the effects of BoNTA on the survival of cutaneous flaps in healthy, diabetic and tobaccoexposed rats. In the group of control animals injected with BoNTA the area of viable skin in the flap was larger than in the control animals. To our knowledge, there are only three publications that investigated the effect of BoNTA on flap viability in healthy animals. In fact, Kim et al. ${ }^{12}$ and Kim et al. ${ }^{13}$ showed better cutaneous flap viability in healthy rats injected with BoNTA. The former adopted a $2 \times 8 \mathrm{~cm}$ random flap and injected 1.5 units of botulinum toxin $\mathrm{A}$ in the central part of the proximal third of the flap at the time of surgery. The latter injected 20 units of botulinum toxin randomly along a $3 \times 10 \mathrm{~cm}$ cutaneous flap seven days before the surgical procedure. The authors observed an increase of $8.3 \%$ and $31 \%$, respectively, in the area of viable skin flap in the animals injected with BoNTA. In addition, the injection of 20units of BoNTA led to no systemic effects. Schweizer et al. ${ }^{11}$ evaluated the effect of BoNTA in two groups of mice, one group injected 24 hours before the procedure and the second group intraoperatively. They presented survival flap areas of $84 \%$ and $88 \%$, respectively, and both were significantly larger when compared with the control group. We chose to inject the animals seven days before operation as it is recognized that the maximum clinical effect of BoNTA is obtained 7-15 days after injection in striated muscle ${ }^{14}$. The survival area observed in animals treated with BoNTA was $34 \%$ larger among the controls. It is noteworthy that increased flap survival was described both in animals injected with BoNTA intraoperatively or $24 \mathrm{~h}$ before the operation. However, the results obtained in rats ${ }^{12,13}$ and mice ${ }^{11}$ are conflicting, regarding which time point of BoNTA injection leads to better responses. Nonetheless, these findings could justify the clinical use of BoNTA injection to improve flap viability even in non-elective situations such as patients submitted to reconstructive procedures with cutaneous flaps due to major trauma or previous acute surgical complications.

Diabetes mellitus is characterized by a series of complications that affect many organs, including microcirculation of cutaneous flaps. Because of long-term incubation with glucose, increments in the chemical modification of proteins lead to the formation of toxic and antigenic glycation end products that decrease endothelial function ${ }^{2,3}$. Despite the large number of clinical studies comparing various reconstructive methods in diabetes, the number of experimental studies evaluating random skin flap survival is limited. In addition, we found no experimental or clinical studies on BontA injection effect on cutaneous dorsal flap viability in diabetes mellitus. In the present study, we evaluated the effect of BoNTA in the viability of cutaneous flap after a 10 -week period of diabetes. According to Serdaroglou et al. ${ }^{19}$, this time interval is sufficient to induce vasculopathy in the experimental model of cutaneous flap ${ }^{18}$. In our sample we found that BoNtA injection increased in 59\% the area of viable skin in diabetic animals. 
Cigarette smoking is associated with endogenous antioxidant depletion, hypercoagulability, and cutaneous vasoconstriction. In addition, tobacco use is associated with a higher incidence of skin necrosis after surgery ${ }^{4}$. Despite the fact that the experimental model of tobacco exposure is well established for respiratory system evaluation ${ }^{20}$, there is only one study dealing with the length of tobacco exposure and its influence on skin flap viability $^{21}$. Biselli's model of tobacco exposure ${ }^{20}$ was employed, once it proved to be feasible and reproducible as confirmed by carboxyhemoglobin measurements. No significant difference was observed in the area of flap necrosis after BoNTA injection in the tobacco-exposed groups. This result is different from Nolan et $a l .{ }^{21}$. They described the same period of exposition but with another equipment. Nolan et $a l .^{21}$ model caused a higher level of the carboxyhemoglobin comparing to our results, $25.2 \%$ versus $14.7 \%$, respectively. Another possible explanation is that the short-time exposure to tobacco did not cause enough endothelial dysfunction that Botulinum toxin could be beneficial.

Concerning the histological results, we observed histological signs suggestive of vasodilation such as larger arterial lumen diameters, external arterial diameters and larger lumen/ wall thickness ratios in the diabetic group that received BoNTA compared to the diabetic+saline group. In the healthy group, although BoNTA improved flap survival, no statistically significant differences were observed regarding arterial diameter. Perhaps BoNTA has an effect on endothelial function and increases blood flow by a mechanism other than vasodilation. Another hypothesis is that BoNTA improves flap survival by neoangiogenesis. In fact, Kim et al. ${ }^{12}$ described a higher number of immature vessels and an increased expression of VEGF (vascular endothelial growth factor) in animals injected with BoNTA. In clinical practice, BoNTA is used as a vasodilator in treating Raynaud's phenomenon. In a study by Neumeister et al. ${ }^{15}$, BoNTA was injected into patients suffering from this condition, with pain and necrotic lesions at the finger extremities ${ }^{22,23}$. After 60 days, all ulcers healed, and $84 \%$ of the patients presented improved vascular flow in their hands. All patients also reported that they no longer had pain ${ }^{15}$. Further studies are necessary to confirm Neumeister's findings.

This study has some limitations. We described here improved skin flap viability in healthy and diabetic rats treated with BoNTA seven days before surgery. Further studies will be conducted to evaluate diabetic animals injected intra-operatively, since it better reproduces practical clinical situations, such as the need of skin flaps in trauma. On the other hand the animals received no treatment for diabetes. The effect of BoNTA injection in animals receiving adequate treatment with insulin should also be investigated. Concerning to tobacco-exposed rats we will perform a long-term exposure to study the effect of BoNTA in this condition.

\section{Conclusions}

Botulinum toxin A have a positive effect on cutaneous flap viability in healthy and diabetic rats on the seventh postoperative day. Furthermore, the results of the histological evaluation of arterial lumen diameter, external arterial diameter, and lumen/ wall thickness ratio suggest a vasodilation effect of BoNTA in the diabetic group. BonTA has no effect on cutaneous flap viability in tobacco-exposed animals on the seventh postoperative day.

\section{References}

1. Gemperli R, Munhoz AM. The influence of type of vascular pedicle occlusion on the viability of skin Island flaps: A postoperative quantitative assessment of flap survival in an experimental model in rats. Acta Cir Bras. 2013;28(7):487-93. PMID: 23842928.

2. Zahiri HR, Lumpkins K, Kelishadi SS, Zhu Y, Medina D, CondéGreen A, Silverman RP, Slezak S, Goldberg NH, Holton LH, Singh DP. Significant predictors of complications after sternal wound reconstruction. A 21-year experience. Ann Plast Surg. 2012;69(4): 439-41. PMID: 22214795.

3. Valentini V, Cassoni A, Marianetti TM, Mitro V, Gennaro P, Ialongo C, Iannetti G. Diabetes as main risk factor in head and neck reconstructive surgery with free flaps. J Craniofac Surg. 2008;19(4):1080-4. PMID: 18650736.

4. Coon D, Tuffaha S, Christensen J, Bonawitz SC. Plastic surgery and smoking: a prospective analysis of incidence, compliance, and complications. Plast Reconstr Surg. 2013;131(2):385-91. PMID: 23358000 .

5. Auclair F, Besnard M, Dupont C, Wepierre J. A new model: bipediculaded dorsal flap of hairless rat for cutaneous blood flow evaluation. Skin Pharmacol. 1989;2 (4):192-203. PMID: 2638868.

6. Campbell SP, Tattelbaum A, Rosenberg M, Chowdary RP, Moss ML, Hugo NE. When does a random flap die? Plast Reconstr Surg. 1989;84(3):484-91. PMID: 2762405.

7. McFarlane MC, Farlane RM, de Young G, Henry RA. The design of a pedicle flap in the rat to study necrosis and its prevention. Plast Reconstr Surg. 1965;35(2):177-82. PMID: 14264468.

8. Camargo CP, Margarido NF, Guandelinei E, Vieira GAB, Jacomo AL, Gemperli R. Description of a new experimental model skin flap for studying skin viability in rats. Acta Cir Bras. 2014;29 (3):16670. PMID: 24626728.

9. Aker JS, Mancoll J, Lewis B, Colen LB. The effect of pentoxifylline on random pattern skin flap necrosis induced by nicotine treatment in the rat. Plast Reconstr Surg. 1997;100(1):66-71. PMID: 9207660.

10. Davies BW, Lewis RD, Pennington G. The impact of vasodilators on random-pattern skin flap survival in the rat following mainstream smoke exposure. Ann Plast Surg. 1998;40(6): 630-6. PMID: 9641282

11. Schweizer D, Schweizer R, Zhang S, Kamat P,Contaldo C, Rieben R, Eberli D, Giovanoli P, Erni D, Plock JA. Botulinum toxin A and $B$ raise blood flow and increase survival of critically ischemic skin flaps. J Surg Res. 2013;184(2):1205-13. PMID: 23651811.

12. Kim TK, Oh EJ, Chung JY, Park JW, Cho BC, Chung HY.The 
effects of botulinum toxin A on the survival of a random cutaneous flap. J Plast Aesthet Surg. 2009;62(7):906-13. PMID: 18436495.

13. Kim YS, Roh TS, Lee WJ, Yoo WM, Tark KC. The effect of botulinum toxin A on skin flap survival in rats. Wound Repair Regen. 2009;17(3):411-7. PMID: 19660050.

14. Berry MG, Stanek JJ. Botulinum neurotoxin A: a review. J Plast Reconstr Aesth Surg. 2012;65(10):1283-91. PMID: 22552262.

15. Neumeister MW, Chambers CB, Herron MS, Webb K, Wietfeldt J, Gillespie JN, Bueno RA, Cooney CM. Botox therapy for ischemic digits. Plast Reconstr Surg. 2009;124(1):191-9. PMID: 19568080.

16. Morris JD, Jobling P, Gibbins IL. Botulinum neurotoxin A attenuates releases of norepinephrine but not NPY from vasoconstrictor neurons. Am J Physiol Heart Circ Physiol. 2002;283(6):H2627-H35. PMID: 12388237.

17. Junod A, Lambert AE, Stauffacher W, Renold AE. Diabetogenic action of streptozotocin: relationship of dose to metabolic response. J Clin Invest. 1969;48(11):2129-39. PMID: 4241908.

18. IIsken T, Serdaroglu I, Ozgentas EThe effects of the pentoxifylline on survival of the skin flaps in streptozotocin-diabetic rats. Ann Plast Surg. 2009;62(4):446-50. PMID: 19325354.

19. Serdaroglu I, Islamoglu K, Ozgentas E. Effects of insulin-dependent diabetes mellitus on perforator-based flaps in streptozotocin diabetic rats. J Reconstr Microsurg. 2005;21(1):51-6. PMID: 15672321.

20. Biselli PJC, Lopes FDTQS, Moriya HT, Rivero DH, Toledo AC, Saldiva PH, Mauad T, Martins MA. Short-term exposure of mice to cigarette smoke and/or residual oil fly ash produces proximal airspace enlargements and airway epithelium remodeling. Braz J Med Biol Res. 2011;44(5):460-8. PMID: 21445523.

21. Nolan J, Jenkins RA, Kurihara K, Schultz RC. The acute effects of cigarette smoke exposure on experimental skin flaps. Plast Reconstr Surg. 1985;75(4):544-9. PMID: 3983255.

22. Stone AV, Koman A, Callahan MF, Eckman DM, Smith BP, Plate JF, Smith TL. The effect of botulinum neurotoxin-A on blood flow in rats: A potential mechanism for treatment of Raynaud's phenomenon. J Hand Surg. 2012;37(4):795-802. PMID: 22386546.

23. Iorio ML, Masdenet DL, Higgins JP. Botulinum toxin A treatment of Raynaud's phenomenon: a review. Semin Arthritis Rheum. 2012;41(4):599-603. PMID: 21868066.
24. Tang Y, Nyengaard JR.A stereological method for estimating the total length and size of myelin fibers in human brain white matter. J Neurosci Methods. 1997;73(2):193-200. PMID: 9196291.

25. Tang Y, Pakkenberg B, Nyengaard JR. Myelinated nerve fibres in the subcortical white matter of cerebral hemispheres are preserved in alcoholic subjects. Brain Res. 2004;1029(2):162-7. PMID: 15542070 .

\section{Acknowledgements}

To Shirley de Sousa, Alexandre Queiros Silva, Joselito Barbosa, and Michele Alberto Marque for technical support.

\section{Correspondence:}

Rolf Gemperli

Disciplina de Cirurgia Plástica-USP

Avenida Dr. Arnaldo, 455

01246-904 São Paulo - SP Brasil

Tel./Fax: (55 11)3062-0415

rgemperli@sti.com.br

Received: May 8, 2015

Review: July 13, 2015

Accepted: Aug 17, 2015

Conflict of interest: none

Financial source: FAPESP (n 2013/02641-3)

${ }^{1}$ Research performed at Laboratory of Microsurgery and Plastic Surgery (LIM-04), Department of Surgery, Universidade de São Paulo (USP), Brazil. Part of PhD degree thesis, Postgraduate Program in Surgical Clinics. Tutor: Rolf Gemperli. 